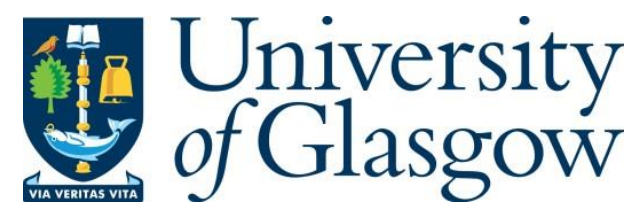

Bouamrane, M.-M., Meiklem, R., Dunlop, M., Kingsmore, D., Thomson, P., Stevenson, K. and Greenwood, S. (2019) Haemodialysis Electronic Patient Portal: A Design Requirements Analysis and Feasibility Study with Domain Experts. In: 2019 IEEE 32nd International Symposium on Computer-Based Medical Systems (CBMS), Cordoba, Spain, 05-07 Jun 2019, pp. 212-216. ISBN 9781728122861.

There may be differences between this version and the published version. You are advised to consult the publisher's version if you wish to cite from it.

$\underline{\text { http://eprints.gla.ac.uk/236429/ }}$

Deposited on: 15 March 2021

Enlighten - Research publications by members of the University of Glasgow http://eprints.gla.ac.uk 


\section{Haemodialysis Electronic Patient Portal: A Design Requirements Analysis and Feasibility Study with Domain Experts}

\author{
Matt-Mouley Bouamrane \\ Usher Institute of Population Health \\ Sciences and Informatics, \\ University of Edinburgh \\ Edinburgh, Scotland \\ FirstName.LastName@ed.ac.uk
}

\author{
Ramsay Meiklem \\ Mark Dunlop \\ Department of Computer \& \\ Information Sciences, \\ University of Strathclyde \\ Glasgow, Scotland \\ FirstName.LastName@strath.ac.uk \\ Sharon Greenwood \\ Social \& Public Policy / Urban Studies \\ University of Glasgow \\ Glasgow, Scotland \\ FirstName.LastName@glasgow.ac.uk
}

\author{
David Kingsmore \\ Peter Thomson \\ Karen Stevenson \\ Glasgow Renal and Transplant Unit \\ Queen Elizabeth University Hospital \\ Glasgow, Scotland \\ FirstName.LastName@nhs.net
}

\begin{abstract}
In 2013, the UK national renal registry established 57,000 adults in the UK were treated for advanced kidney failure, $23,683(42 \%)$ receiving haemodialysis. Haemodialysis patients face some of the highest treatment burden in the National Health Service (NHS) and are among the most 'expensive' to treat. In addition, patients endure complex treatment trajectories. In this study, we have sought to gather and synthesise the opinion of clinical and Human Computer Interaction (HCI) domain experts $(n=9)$ to establish a set of initial design requirements in order to test the feasibility of developing a digital aid (i.e. electronic haemodialysis patient portal) to support patients in the course of their treatment. Expert feedback was gathered by means of interviews and focus groups in order to instruct design requirements for a haemodialysis patient portal.
\end{abstract}

Keywords: advanced chronic kidney disease; patient portal; design requirements; experts study

\section{INTRODUCTION}

Chronic kidney disease is a substantial global health burden with a very high associated economic cost to health systems [1]. It is a very significant independent risk factor for further morbidities, including cardiovascular diseases, premature mortality, and a substantial decrease in quality of life (QoL) [1-3].

Furthermore, treatment options and trajectories for patients with advanced chronic kidney diseases (ACKD) are complex and subject to important and possibly unjustified variations. A recent mixed-methods national appraisal of haemodialysis (HD) vascular access (VA) provision in Scotland highlighted substantial variation in the use of different VA modalities between Scottish renal units [4] and patients reported frustration and dissatisfaction with their personal experiences of treatment. The study recommended better staff education, multidisciplinary focus on patient care, clinical pathway optimisation and improved measurements of clinical and patient outcomes to improve VA service quality and facilitate safer and more effective, patient-centred care [4].

In this study, we have sought to address some of these recommendations, focusing on developing an electronic patient portal for patients with ACKD. In the initial stages of the ACKD portal development, we have sought to gather design requirements from domain experts (clinical and Human Computer Interaction (HCI)). This study focuses on reporting results from this design requirements elucidation phase and is structured as follows: background information on chronic kidney disease and HD in section II, methods in section III and study results in section IV.

\section{BACKGROUND}

Haemodialysis (HD) is a life-prolonging medical procedure carried out when kidneys no longer function normally, such as in the case of ACKD [5]. The patient's blood is mechanically filtered by a HD machine, removing excess fluid and waste products from the blood - a task conducted by the kidneys when functioning normally. Treatment is typically performed three times a week, sessions lasting four to five hours. HD requires a vascular access (VA) method for cannulation (i.e. insertion of 'tube' to access blood vessels). There are various VA methods currently used in clinical practice, and make for complex patient pathways and complications of VAs are also common [6-7].

Studies have shown that HD places a high treatment burden on patients and contributes significantly to lower QoL [1-3]. A recent qualitative study found that UK patients on HD experienced a range of fluctuations in cognitive/physical well-being across the HD cycle, severe restrictions arising from the treatment schedule, and that HD had substantial emotional impact on patients, their families and social relationships [8]. These findings suggest that HD patients need additional support to manage their care using a holistic and patient-centred approach. 
Electronic clinical portals have been shown to be very useful tools for continuity of care and multidisciplinary management of perioperative patients [9-11].

\section{METHODS}

\section{A. Study Participants}

This study was conducted with the Queen Elizabeth University Hospital (QEUH) in Glasgow. This study was approved by the institutional ethics board at the University of Strathclyde in June 2018. The QEUH is a modern 1,677-bed acute hospital and hosts the largest renal service in Scotland. Nine domain experts were recruited and participated in a series of individual semi-structured interviews and "thinkaloud" focus group sessions.

The experts who participated are described in Table 1.

Table 1. Description of Domain Experts

\begin{tabular}{clc}
\hline ID & Profession & Sex \\
\hline E1 & $\begin{array}{l}\text { Consultant, Vascular and Renal Transplant } \\
\text { Surgery }\end{array}$ & F \\
E2 & Consultant, Transplant and General Surgery & M \\
E3 & Consultant Nephrologist & M \\
E4 & Locum Consultant, Clinical Research Fellow & M \\
E5 & $\begin{array}{l}\text { Consultant Nephrologist, Scottish Patient Safety } \\
\text { Program Fellow }\end{array}$ & M \\
E6 & $\begin{array}{l}\text { Sociology Research Assistant, Qualitative } \\
\text { Methods and Quality of Life }\end{array}$ & F \\
E7 & $\begin{array}{l}\text { Senior Academic ('associate professor' level), } \\
\text { Digital Health }\end{array}$ & M \\
E8 & $\begin{array}{l}\text { Senior Academic ('associate professor' level), } \\
\text { Digital Health }\end{array}$ & F \\
E9 & $\begin{array}{l}\text { Senior Academic ('associate professor' level), } \\
\text { Human Computer Interaction (HCI) }\end{array}$ & F \\
\hline
\end{tabular}

\section{B. Data Collection}

All participants $(n=9)$ provided consent and participated in a total 12 interviews and focus groups. We initially conducted one-to-one semi-structured interviews $(n=6)$ with clinical experts (E1-E6) to elucidate existing issues or limitations with the care of patients on HD and gather the portal system requirements. The semi-structured interviews allowed participants to begin discussions about issues and topics related to ACKD patient care.

Once an initial patient portal prototype was developed, a set of focus groups was then conducted $(n=6)$ to gather iterative expert feedback on the prototype, where specific functionalities were discussed in depth and then subsequently revised and updated prior to the next focus groups. All participants from the original interviews were invited back to provide feedback in these sessions along with $(n=3)$ new participants regarded as experts in Digital Health or HCI (E7E9). Focus groups were conducted with up to 4 participants at a time, with 2 researchers present. The interviews and focus groups lasted an average time of 55 minutes and were all held on site (i.e. dedicated meeting room at QEUH) between June and November 2018.
During the 'think-aloud' focus groups, participants could interact with the prototype, speaking aloud any thoughts or opinions as they completed tasks and investigated the prototype. Guidance and assistance were available if requested but participants were encouraged to explore the prototype independently and talk openly at the start of all sessions, with researchers allowing time for pause in discussion.

\section{Data Analysis}

The audio of all interviews and focus groups was digitally recorded with consent of participants and transcribed for analysis.

We analysed the data using a health information systems quality assessment framework for coding the transcripts of interviews [12]. The framework is derived from DeLone \& McLean's model of quality in information systems [13].

The framework comprises the following 6 dimensions: (i) eHealth information system quality; (ii) information quality; (iii) information usage; (iv) user satisfaction; (iv) individual impact; and (vi) organisational impact. Through iterative amalgamation of related codes, new distinct themes emerged which we grouped into those described in section IV.A.

\section{RESULTS}

\section{A. Thematic Analysis of Requirements for a Haemodialysis Patient Portal}

T1: Information System Quality: Importance of Good and Simple Design.

The clinicians interviewed confirmed HD patients faced significant treatment burden, and attended the hospital frequently and for significant time. They expected that patients would be keen to engage with a digital system that recorded their experiences, particularly during treatment as this could be a positive distraction during a lengthy procedure. They emphasised the need for a high-quality and simple design nevertheless, to maintain the patients' engagement over time while catering for the wide range of potential users, with varying levels of health and technical literacy present across the patient population. For example, it was stated by E1: "I think the reason people will do it, is if it's easy and pleasant to interact with. They won't do it for points or prizes or anything else, they'll do it because it's simple, easy and they feel they've bought into it and somebody's interested."

\section{T2: Information Quality: Inadequate or Missing Data.}

All clinical experts interviewed raised the issue that the current information systems in use provided incomplete and limited access to patient data and this prevented them from understanding how effective treatments provided were. It was noted that while some information was being recorded during the clinical encounters at the hospital, clinicians had limited insight into treatment burden in daily life and other aspects of 'disease management' once patients left after HD sessions. 
Examples of these issues included: no QoL data currently available in hospital information systems; location of VA cannulation during HD not appropriately recorded or available; recording of clinical events not always complete; no means of investigating impact of treatment decisions on patient QoL; staff occasionally rely on patients recalling treatment information (e.g. previous VA cannulation location). Missing and incomplete data was described by E2: "...That's data that is no lon-not recorded anywhere at the minute, on anything. Some of the clinic events may or may not be, hospital admissions or complications... Like if you have a bad cannulation or y'know, it hurts, it's not recorded anywhere. So, some of its recorded, some of it isn't at the minute..."

T3: Information Usage: Measuring Care Quality and Generating New Insights on Treatments.

Immediately related to the previous theme, this one regards the usage of information which would be provided by a more comprehensive capture of patient data. It was thought that this would enable better understanding of the quality of treatments and provide new insight by cross-referencing the new data with that already captured by existing hospital formation systems, as suggested by E1: "Actually, we can link that [data recorded by proposed system] by date by looking at their other stuff [existing patient data]- which is actually probably better...yeah so in terms of... - it's about being able to put them in the same place or link them at some point."

T4: User Satisfaction: Simplifying and Clarifying Treatment Options for Patients.

Clinicians confirmed patients were often confused by the range of treatment options available to them and this could lead to frustration and dissatisfaction with care they received noted previously [4]. This was partly blamed on the lack of a standardised overview of treatment options and care pathways. It was suggested by E3 that functionalities in a patient portal that guided patients through treatment options could lead to an improvement in patients' understanding and satisfaction with their care: "They don't feel like they're the expert in their care...It's about health literacy...A lot of the patients, they know what they're told by consultants, but they don't - They're just rehashing what they've been told. I've found patients are quite resistant to advise other patients... a lot of the time they would say, 'Oh, I'm not placed to advise anyone...",

T5: Individual Impact: Improving Patients Outcomes Measures.

Clinicians emphasised better information capture and analysis as a driver for care quality improvement and better outcomes for patients. A key argument in support of a patient portal is the potential to allow tailored VA and treatment options depending on how each patient responds, which is very much aligned to current strategic priorities of 'personcentred' care and 'personalised medicine'. For example, E6 stated: "... what you want to see is, when the [vascular] access is good, is it very very good? And when it's bad just how awful was it? And you can then say, 99 times out of a 100, it'll run fine. And you'll have a very, very good qualitative argument..."

T6: Organisational Impact: Developing Optimal Care Pathways.

In order to optimise patient care pathways, the benefits of a data-driven approach were discussed. Using new data sources such as patient outcomes measures (T5), clinicians would be able to support decisions and redesign regarding services and treatments. E2 discussed this in regards to future clinical trials and studies: "Ultimately it's about trying to pick out in a trial cohort where you've got two alternative strategies, what's worse for a patient... where you hope to see a difference is potentially the impact of different types of [vascular] access..."

\section{B. Formal Design Requirements for a Haemodialysis Patient Portal}

The thematic analysis was used to develop a formal set of 'high-level' design requirements for the patient portal, which were discussed with the experts before the development of an initial prototype. The set of requirements is provided in Table 2 , including reference to relevant themes and issues analysed during the qualitative data analysis.

Table 2. High-level formal requirements for Haemodialysis Patient

\begin{tabular}{lll}
\multicolumn{2}{c}{ Portal } & Themes \\
\hline 1 & Capture of Quality of Life (QoL) measure & T2, T5 \\
2 & Capture of Important Clinical Events & T2 \\
3 & Capture of Vascular Access data & T2 \\
4 & Data Linkage between various data sources & T3 \\
5 & Data Processing: providing new insight into & T3, T5, T6 \\
& treatment impact & T3, T4, T5 \\
6 & $\begin{array}{l}\text { Interactive treatment guide \& patient care } \\
\text { pathways }\end{array}$ & T1, T4 \\
7 & $\begin{array}{l}\text { System should be well designed, have high } \\
\text { accessibility and usability to aid users with }\end{array}$ & \\
& varying ability and experience &
\end{tabular}

\section{Haemodialysis Patient Portal Prototype Development \& Expert Feedback}

A prototype patient portal system was developed using the formal design requirements and themes that emerged during the focus groups are described in detail and were used to further refine the portal requirements. Screenshots of the user interface of the prototype produced can be seen in Fig. 1. We used the thematic analytical framework of Information Systems Quality [12-13] described in section III.C to analyse the feedback collated during focus groups. During the focus 

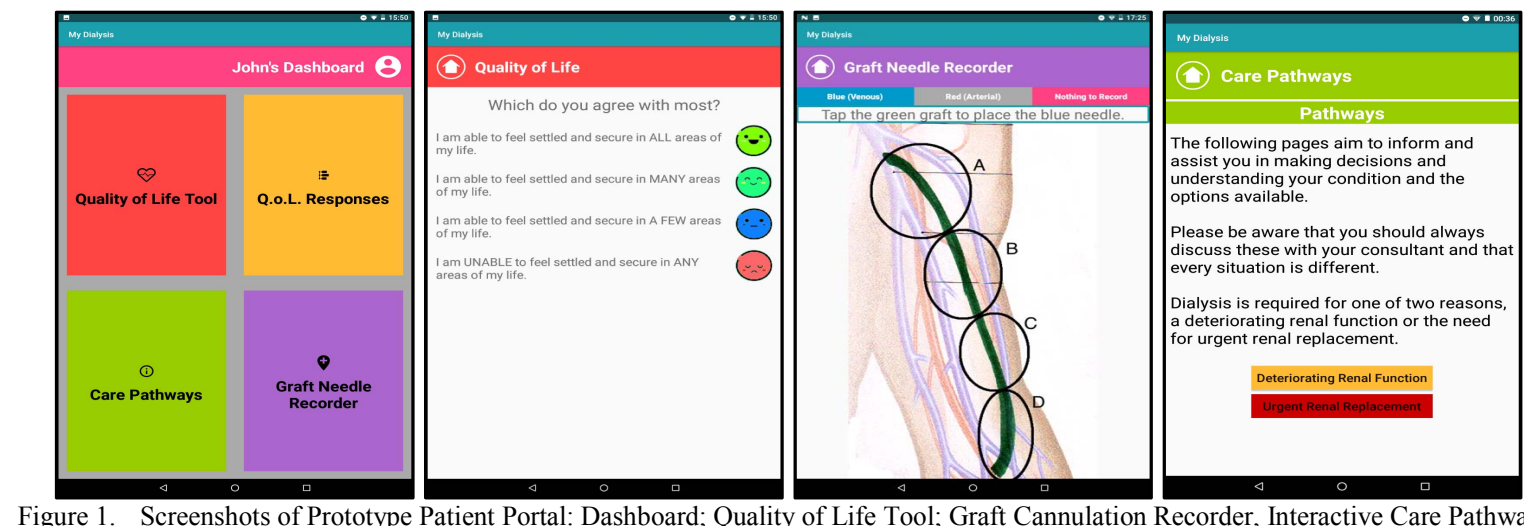

Figure 1. Screenshots of Prototype Patient Portal: Dashboard; Quality of Life Tool; Graft Cannulation Recorder, Interactive Care Pathways.

groups, the experts provided feedback on: information added value, user interaction and accessibility.

\section{Information Usage: Information Added Value.}

The format and analysis of potential data captured by the patient portal was discussed in depth. Additional suggestions were made for implementing further 'analytic' functionalities, including: sorting and filtering of data by date, level of QoL responses, clinical events, aspect of QoL, etc.; display data as timeline or graph over time, to highlight peaks, troughs and other patterns; display previous VA cannulation sites (unsuitable for current cannulation) during capture of cannulation data; and display QoL data alongside clinical events data, described by E2: "So what I'm looking for, there has to be an event as well...has anything happened, that tells you something... [...] And the idea is presumably like if you have something like "access clots up" your Quality of life, $y^{\prime k n o w}$ your Quality of Life plummets with that, $y^{\prime}$ know. And then it changes- we have to tailor, correlate that with clinical events, that's absolutely key."

User Satisfaction: Insufficient Interaction Feedback.

It was noted that the prototype did not offer sufficient feedback in some tasks, which was confusing for users. Experts suggested "pop-up" feedback could be implemented in order to communicate system status for functionalities such as the graft cannulation recorder or the QoL tool. Other suggestions included implementing audio and visual feedback and cues, such as 'click' sounds with users touching interface elements or animations drawing attention to important elements of the interface.

User Satisfaction: Accessibility of Patient Portal.

Experts provided positive feedback on the 'minimalist' interface which promoted efficient learning of the core functions of the system. Providing support for visual impairments was discussed in depth, as partial sight loss or complete blindness are common comorbidities associated with ACKD patients [16].

For example, E8 discussed some accessibility measures: "That's what I was going to say, maybe more pictures and
links...Like we can't just assume that people can read everything and sometimes its videos and things like that for people...it's so much more accessible... [...] Could you have, link in the future, voice recognition?...it'll ask you the question and you answer it, nothing to do with text."

Suggestions for additional accessibility functionalities included: text-to-speech audio output for visually impaired users; voice recognition for user input; recording of user settings or preferences (background colour, text font size, colour, etc.); adaptive user interface layouts to support user interaction with single available hand during HD treatment; alternative media alongside patient care pathways text information (e.g. images, audio, videos, etc.).

\section{CONCLUSION}

To the best of our knowledge, this study is the first to elucidate and define a set of formal requirements for the implementation of a clinical portal for ACKD patients/patients receiving HD treatment. While some requirements are likely generic to patient portal systems in general, some unique disease specific requirements were identified, including the need for VA specific QoL measure and the need to capture VA cannulation (Req. 1, 3). Other requirements highlighted that - although the functionalities could be construed as more generic - the real added value came from condition specific content (i.e. interactive treatment guides and patient care pathways, Req. 6) and condition specific analytics to assess disease trajectories and treatment impact (Req. 2-5). This suggests for high treatment burden conditions such as HD, capturing the idiosyncratic requirements inherent to the condition is essential for successful deployment of a patient portal designed for this patient group. We intend to test this hypothesis by conducting a follow-up study with HD patient groups in the near future.

\section{ACKNOWLEDGMENT}

This research was funded through a Medical Research Council 'Confidence in Concept' Grant (2018-2019). 


\section{REFERENCES}

[1] N. Hill et al., "Global Prevalence of Chronic Kidney Disease - A Systematic Review and Meta-Analysis", PLOS ONE, vol. 11, no. 7, p. e0158765, 2016. Available: 10.1371/journal.pone.0158765.

[2] S. Fukuhara et al., "Health-related quality of life among dialysis patients on three continents: The Dialysis Outcomes and Practice Patterns Study", Kidney International, vol. 64, no. 5, pp. 1903-1910, 2003. Available: 10.1046/j.15231755.2003.00289.x

[3] J. Cleary and J. Drennan, "Quality of life of patients on haemodialysis for end-stage renal disease", Journal of Advanced Nursing, vol. 51, no. 6, pp. 577-586, 2005. Available: 10.1111/j.1365-2648.2005.03547.x.

[4] S. Oliver et al., "A national appraisal of haemodialysis vascular access provision in Scotland", The Journal of Vascular Access, vol. 18, no. 2, pp. 126-131, 2017. Available: 10.5301/jva.5000651.

[5] P. Stenvinkel, "Chronic kidney disease: a public health priority and harbinger of premature cardiovascular disease", Journal of Internal Medicine, vol. 268, no. 5, pp. 456-467, 2010. Available: 10.1111/j.1365-2796.2010.02269.x.

[6] E. Aitken, P. Thomson, L. Bainbridge, R. Kasthuri, B. Mohr and D. Kingsmore, "A randomized controlled trial and costeffectiveness analysis of early cannulation arteriovenous grafts versus tunneled central venous catheters in patients requiring urgent vascular access for hemodialysis", Journal of Vascular Surgery, vol. 65, no. 3, pp. 766-774, 2017. Available: 10.1016/j.jvs.2016.10.103.

[7] H. Feldman, S. Kobrin and A. Wasserstein, "Hemodialysis vascular access morbidity", Journal of the American Society of Nephrology, vol. 7, no. 4, pp. 523-535, 1996.

[8] D. Jones, K. Harvey, J. Harris, L. Butler and E. Vaux, "Understanding the impact of haemodialysis on UK National Health Service patients' well-being: A qualitative investigation", Journal of Clinical Nursing, vol. 27, no. 1-2, pp. 193-204, 2017. Available: 10.1111/jocn.13871.

[9] M. Bouamrane and F. Mair, "Implementation of an integrated preoperative care pathway and regional electronic clinical portal for preoperative assessment", BMC Medical Informatics and Decision Making, vol. 14, no. 1, 2014. Available: 10.1186/1472-6947-14-93.

[10] M. Bouamrane and F. Mair, "A Study of Information Management in the Patient Surgical Pathway in NHSScotland", Studies in health technology and informatics: MedInfo, no. 192, pp. 557-561, 2013.

[11] M. Bouamrane, A. Adeyemo, M. McGee-Lennon and S. Schraag, "Patient Preoperative Web Portal for Remote, Selfcompleted Assessment \& Triage: a Feasibility Study", in Proceedings of 2015 BCS Health Informatics Scotland Conference, BCS eWic, Electronic Workshops in Computing, 2015

[12] M. Bouamrane, F. Mair and C. Tao, "An overview of electronic health information management systems quality assessment", in Proceedings of the 2nd international workshop on managing interoperability and complexity in health systems, ACM, 2012.
[13] W. DeLone and E. McLean, "Information Systems Success: The Quest for the Dependent Variable", Information Systems Research, vol. 3, no. 1, pp. 60-95, 1992. Available: 10.1287/isre.3.1.60.

[14] M. Bouamrane and F. Mair, "A qualitative evaluation of general practitioners' views on protocol-driven eReferral in Scotland", BMC Medical Informatics and Decision Making, vol. 14, no. 1, 2014. Available: 10.1186/1472-6947-14-30.

[15] M. Bouamrane and F. Mair, "A study of general practitioners' perspectives on electronic medical records systems in NHSScotland", BMC Medical Informatics and Decision Making, vol. 13, no. 1, 2013. Available: 10.1186/1472-694713-58.

[16] S. Bansal, A. Ansons and M. Vishwanath, "Hypotensioninduced blindness in haemodialysis patients", Clinical Kidney Journal, vol. 7, no. 4, pp. 387-390, 2014. Available: $10.1093 / \mathrm{ckj} / \mathrm{sfu} 036$. 